\title{
Investigation of structural differences of silica, silver and iron nanoparticles on the proliferation of human lung cancer
}

\begin{abstract}
Lung cancer, one of the critical respiratory system diseases, has the highest morbidity rate $(14.3 \%)$ among men and third in women $(8.4 \%)$ worldwide. Severe side effects and ineffectiveness of chemotherapeutic drugs lead to the investigation of new functional agents. One of the latest strategies against the proliferation of cancer is using nanoparticles. On the other hand, the natural composition of those particles significantly affects their efficacy. This study aimed to investigate the effects of the structural differences of silver, iron, and silica nanoparticles on lung cancer cells. All nanoparticles were synthesized with an average size of $40 \mathrm{~nm}$, and their particle sizes were characterized by Dynamic Light Scattering (DLS) and Transmission electron microscopes (TEM). The cell viability and cytotoxic potential of nanoparticles were investigated using the Alamar Blue reagent. The ratio of the alive and dead cells was determined by the Automated Cell Counter using Trypan Blue. Localization of nanoparticles in the cells was visualized floresans microscope. Within the nanoparticles, the silica nanoparticle (SiNPs) showed the highest cytotoxicity in the lung cancer cell line with an $\mathrm{IC}_{50}$ value of $151.3 \mu \mathrm{g} / \mathrm{mL}$ in a dose-dependent manner. Neither FeNPs nor AgNPs showed significant toxic effects on the proliferation of A549 cells $(>250 \mu \mathrm{g} / \mathrm{mL})$. SiNPs were localized mainly in the cytoplasm. SiNPs have seen higher catalytic activity than AgNPs and FeNPs. The functionalization of the surface of silica nanoparticles and the ability to bind functional groups or drug candidates both on the surface and inside make them an essential agent in cancer treatments.
\end{abstract}

Keywords: nanoparticles, lung cancer, cytotoxicity, structural difference, SiNPs, AgNPs, FeNPs
Volume 9 Issue 4 - 202|

\author{
Sureyya Erturk, Irem Sobaci, Irem Bereket, \\ Sadik Seker, Gamze Polat, Serdar Karakurt \\ Department of Biochemistry, Faculty of Science, Selcuk \\ University, Turkey
}

\begin{abstract}
Correspondence: Serdar Karakurt, Department of Biochemistry, Faculty of Science, Selcuk University, Konya, Turkey, Tel +903322233889, Email kserdar I@yahoo.com
\end{abstract}

Received: July 16, 202I | Published: July 26, 2021

\section{Introduction}

Cancer is a complex disease that occurs with the uncontrolled division and proliferation of cells and is influenced by genetic and environmental conditions. ${ }^{1}$ Today, breast cancer is the most common type of cancer in women and lung cancer in men. ${ }^{2}$ The incidence of lung cancer, which is the most common respiratory system disease, is in the first place in men and third in women. Mortality in lung cancer comes first in both genders. ${ }^{3}$ The most important reason for the high mortality is the difficulties encountered in early diagnosis. Despite the different drug effects, the therapeutic treatments or the combined therapeutic treatment formulation have had no impact on reducing tumorigenesis and mortality. ${ }^{4,5}$ Commonly used treatment modalities are bone marrow transplant, radiotherapy, and chemotherapy. It has been observed that these traditional treatment methods in early diagnosis and treatment are sometimes insufficient both in diagnosis and treatment. With its insufficiency, it causes severe side effects and causes adverse effects on human life. The tendency to new treatment methods should be increased by using new technologies developed. Nanotechnology, which can be considered one of the best examples of developing technology, is a new science that carries out ambitious studies in health. Nanoparticles from nanostructures; consist of macromolecular materials and can be used therapeutically, especially in the controlled delivery of drugs to the desired area. ${ }^{6}$ Nanoparticles are used as anticancer agents; It has been seen that they can be used for purposes such as reducing toxicity, improving in vivo and in vitro stability. In this way, while the intracellular concentrations and toxic effects of drugs in cancer cells can be increased, their harmful effects on healthy cells can be minimized. ${ }^{7,8}$ Silver nanoparticles (AgNP), which have an important place among nanoparticles with proven biological activities, have been proven to have cytotoxic effects in prokaryotic cells and eukaryotic cells. ${ }^{9}$ Because AgNPs containing hybrid molecular units have outstanding biocompatibility for therapeutic environments, they are used in the design of drug delivery systems sensitive to optical, thermal and $\mathrm{pH}$ modulations to target malignancies, inflammatory and infectious disorders. ${ }^{10}$ AgNPs induce ROS accumulation in cancer cells, which leads to the inflammatory response, degradation, and subsequent destruction of mitochondria. ${ }^{11}$ AgNPs have an effective toxic effect on many types of cancer. However, the different effects of the nanoparticles used, especially in various sizes, have different effects on the cells. ${ }^{12}$ Silica nanoparticles are among the most popular nanoparticle types among researchers due to their superior properties with functional versatility. ${ }^{13}$ Silica nanoparticles are one of the most preferred nanoparticles because they can be easily synthesized, functionalized in many different ways, biocompatible, stable in different conditions, and easy to apply. ${ }^{14}$ Silica nanoparticles are highly effective in cancer treatment and transport of colloids to tumor sites by blood. However, the different sizes, shapes, and surface charges of the nanoparticles used have different effects on the cells. ${ }^{15}$ FeNPs have emerged as a new generation of targetspecific contrast agents. Magnetic FeNPs are much more effective than many nanoparticles because their magnetic properties can be changed by controlling the size of the core and coating surface. ${ }^{16}$ More importantly, FeNPs have long-term blood retention, biodegradability, and low levels of toxicity. ${ }^{17,18}$ For the development of tumor-targeted FeNPs, different ligands such as antibodies, peptides, and small 
molecules targeting the relevant receptors, which are highly expressed in tumor cells, are usually conjugated to the surface of FeNPs. ${ }^{19}$ Iron nanoparticles are used not only in cancer diagnosis and diagnosis but also in imaging. ${ }^{20}$ Hence, this study aims to investigate the effects of nanoparticles of the same size but different structural properties on human lung cancer.

\section{Material and methods}

\section{Chemicals}

Tetraethyl orthosilicate $(\geq 99.0 \%(\mathrm{GC}))$, ethyl alcohol $(99.8 \%)$, Ammonia solution (25\%), Polyacrylic Acid, Iron(III) chloride Palladium(II) chloride solution ( $5 \%$ in $10 \% \mathrm{HCl}$ ), Sodium borohydride $(\geq 98.0 \%)$, Aceton (HPLC, $\geq 99.9 \%$ ) , tri-Sodium citrate dihydrate, Silver nitrate $(\geq 99.0 \%)$, Sodium Hydroxide were purchased from Sigma-Aldrich (USA). Human lung carcinoma cells (A549), Fetal Bovine Serum (FBS), and Dimethylsulfoxide (DMSO) were obtained from American Type Culture Collection (ATCC). All materials used in the experiments were of standard analytical grade from SigmaAldrich and used without further purification.

\section{Synthesis of nanoparticles}

Silica nanoparticles were synthesized by the sol-gel method using tetraethoxysilane (TEOS) as a silica source in a solvent environment. First, Ammonia and Ethanol (Et-OH) were mixed in a mixer at room temperature; It was added slowly with a mixture of TEOS and Et-OH. All these synthesis processes in the fume hood took 24 hours. After 24 hours, the synthesized silica nanoparticles were taken from the mixer and centrifuged in $1.5 \mathrm{~mL}$ centrifuge tubes at $14000 \mathrm{xg}, 20^{\circ} \mathrm{C}$, for 1 hour. The supernatant formed at the end of centrifugation was removed, and the solvent Et-OH was added to the pellet and kept in an ultrasonic bath for 30 minutes. After washing with Et-OH, SiNPs was centrifuged again at $14000 \mathrm{xg}, 20^{\circ} \mathrm{C}$ for 1 hour, and these processes were repeated 3 times. Finally, the purified SiNPs were allowed to dry overnight. ${ }^{21}$ Next, iron nanoparticle synthesis was carried out using a high $\mathrm{pH}$ disintegrant. Polyacrylic acid and $\mathrm{FeCl}_{3}$ were mixed, then $\mathrm{PdCl}_{2}$ and $\mathrm{NH}_{4} \mathrm{OH}$ were added and mixed in a magnetic stirrer. After the bubble formation was completed by adding $\mathrm{NaBH}_{4}$ to the mixture obtained, acetone was added to separate the nanoparticles, and the nanoparticles were separated with a magnet. After washing 3-4 times with distilled water, it was dried at $-20^{\circ} \mathrm{C}$. Silver nanoparticles were synthesized using $\mathrm{NaBH}_{4} \cdot \mathrm{NaBH}_{4}$ was used as the first reducing agent, and trisodium citrate (TSC) was used as the second reducing and stabilizing agent. TSC and $\mathrm{NaBH}_{4}$ were mixed for 30 minutes at $60^{\circ} \mathrm{C}$, and the temperature was brought to $90^{\circ} \mathrm{C}$ after $\mathrm{AgNO}_{3}$ was added. When the solution reached $90^{\circ} \mathrm{C}$, the $\mathrm{pH}$ was adjusted to 10.5 using $0.1 \mathrm{M} \mathrm{NaOH}$ and stirred for 20 minutes. When the color change was observed at the end of the mixing process, the solution was cooled to room temperature. It was centrifuged at $12.000 \mathrm{rpm}$ for 15 minutes for unreacted reductants and washed 3 times with deionized water.

\section{Transemission electron microscopy (TEM)}

The size and shape of the nanoparticles (NP) were determined using a scanning electron microscope operating at $3 \mathrm{kV}$.

\section{Dynamic light scattering (DLS)}

After the nanoparticles were kept in the ultrasonic bath for 30 seconds, the particle sizes of the NPs were determined under a $632.8 \mathrm{~nm}, 35 \mathrm{~mW}$ light source with the help of Dynamic Light Scattering (DLS).

\section{Cell culture and treatment}

Human lung carcinoma cells, A549, were cultured in EMEM medium supplemented with $10 \%$ FBS (fetal bovine serum), 100 unit/mL penicillin/ streptomycin Penicillin-streptomycin, and $2 \mathrm{mM}$ L-glutamine at $37^{\circ} \mathrm{C}$ under $5 \% \mathrm{CO}_{2}$ and $95 \%$ air. The cells were detached with $0.25(\mathrm{w} / \mathrm{v})$ trypsin-EDTA solution from the cell culture plate when they reach $\% 80$ confluence. To investigate the cytotoxicity of SiNPs, AgNPs, and FeNPs, $1 \times 10^{4}$ cells per well were seeded into 96well plate and incubated for $24 \mathrm{~h}$. After removing the growth medium, the cells were treated with various concentrations of nanoparticles ranging from $0-250 \mu \mathrm{g} / \mathrm{mL}$. After $48 \mathrm{~h}$ of treatment, the cell viability was determined via Alamar Blue Assay (Invitrogen, Thermo Fischer Scientific, Waltham, MA, USA) using a spectrophotometer (Thermo, MultiScanGO, USA), and the viable-dead cell ratios were determined using $0.04 \%$ "Trypan Blue" using an Automatic Cell Counting Device. ${ }^{22}$

\section{Cell imaging}

To determine the intracellular localization of the synthesized nanoparticles, the NPs were treated with A549 cells at the concentration of the $\mathrm{IC}_{50}$ values. First, cells were inoculated at $10^{5}$ cells/well in 24well plates suitable for glass-bottom black fluorescence studies and incubated for 24 hours at $37^{\circ} \mathrm{C}, 5 \% \mathrm{CO}_{2}$. Then, the cells were treated with nanoparticles and incubated for 45 minutes. After incubation, the cells were washed 3 times with $10 \mathrm{mM}$ PBS and examined with a ZOE fluorescence microscope (Bio-Rad, USA).

\section{Statistical analyses}

Statistical analyses of the data were evaluated by GraphPad Prism 8.0 software. The data were represented as mean $\pm S D, n=12$. The obtained results were analyzed using one-way ANOVA and Student's $\mathrm{t}$-test. The significance level was set to $\mathrm{p}<0.05$.

\section{Results and discussion}

Nanoparticles showed an important role in diagnosing and treating lung cancer due to their targeted drug delivery, bioavailability, intestinal absorption, and solubility. ${ }^{23}$ Nanoparticles have different effects on lung cancer cells due to their structural differences. ${ }^{24}$ Nanoparticles used to diagnose and treat lung cancer can be structurally classified as magnetic nanoparticles, polymer nanoparticles, liposomes, solid lipid nanoparticles, metal nanoparticles, virus nanoparticles, and quantum dots. ${ }^{25} \mathrm{FeNP}$ and AgNP used in the study are examples of magnetic and metal nanoparticles. Magnetic nanoparticles also have advantages, such as directing them to the tumor site with an external magnetic field. ${ }^{26}$ Magnetic nanoparticles should be $<200 \mathrm{~nm}$ to prevent spleen and liver filtration and $>10 \mathrm{~nm}$ to prevent rapid renal filtration. ${ }^{27} \mathrm{In}$ this study, DLS analysis was performed for each synthesized nanoparticle, and the nanoparticle size and the densities of the NPs were calculated. DLS analysis of AgNP demonstrated that the density is $100 \%$ between 10 and $100 \mathrm{~nm}$. Therefore, the NP size was measured at $40 \mathrm{~nm}$. As a result of the analysis for FeNP, the density between 10 and $100 \mathrm{~nm}$ is $5.6 \%$, and the NP size is around $48 \mathrm{~nm}$. Finally, the DLS-analyzed SiNP has a density of about $24 \%$ between 10 and $100 \mathrm{~nm}$, and the NP size was measured at about 40nm (Figure 1).

The cytotoxic effects of silica, iron, and silver nanoparticles on A549 cells, a metastatic cell line from human lung cancer cell lines, were investigated. Cells were treated with nanoparticles, and percent cell viability versus $\log$ concentration graphs was drawn sigmoidal. The $\log$ (inhibitory) vs. sigmoidal graphs were obtained, 
and normalized response-Variable slope analysis was performed with GraphPad Prism 8.0 software, and $\mathrm{IC}_{50}$ values were calculated. SiNPs showed significant cytotoxicity on the viability of A549, and the $\mathrm{IC}_{50}$ value was determined as $151.3 \mu \mathrm{g} / \mathrm{mL}$. Neither FeNPs nor AgNPs showed significant toxic effects on the proliferation of A549 cells $(>250 \mu \mathrm{g} / \mathrm{mL})$. The results obtained from the data obtained from the sigmoidal graphs showed that the percentage (\%) of cell viability versus the log concentration graph in cancer cells treated with silver and iron nanoparticles continued to survive at a rate of $80 \%$. Still, the rate reached in silica nanoparticles was $60 \%$, and the cell viability could be maintained at this rate. Chauhan et al. observed that in the cells treated with FeNP in the first 24 hours, cell viability was found to be $85 \%$ at a concentration of $10 \mu \mathrm{g} / \mathrm{mL}$ and the cell viability decreased below $50 \%$ after $200 \mu \mathrm{g} / \mathrm{mL}$ at the end of 48 hours. ${ }^{28}$ In the study conducted with Superparamagnetic Iron Oxide Nanoparticles (SPION), SPION and SPION coated $\mathrm{SiO}_{2}$ nanoparticular toxicity in A549 cells increased 30-fold compared to the control group. ${ }^{29}$
Metal nanoparticles are agents with a wide range of uses. It is used in radiotherapy for augmentation and diagnostic imaging at the beginning of its biomedical usage areas. Their physicochemical properties, easy modification of particle surfaces, biocompatibility create an anticancer effect and suppress tumor progression. ${ }^{30-33} \mathrm{AgNPs}$, on the other hand, are nanoparticles with many biological properties, especially in the field of halth, with antimicrobial and anticancer effects. He et al. demonstrated that AgNPs dose-dependently inhibited the proliferation of various human cancerous cells including, H1299 (Lung cancer), VcaP(Prostate cancer), and BxPC-3 (pancreas cancer) cells with an $\mathrm{IC}_{50}$ value of $5 \mu \mathrm{g} / \mathrm{mL}, 87.33 \mu \mathrm{g} / \mathrm{mL}$, and $38.9 \mu \mathrm{g} / \mathrm{mL}$, respectively. ${ }^{34}$ Besides, size differences also affect the cytotoxicity of AgNPs on human lung cancer. Gliga et al. (2014) proved that small-size AgNPs (10nm) have the highest toxicity within the other sizes $(40 \mathrm{~nm}, 50 \mathrm{~nm}, 75 \mathrm{~nm}){ }^{35}$ This can be explained with the highest intracellular Ag concentration called as "Trojan horse' effect." That was correlated with our findings (Figure $2 \& 3$ ). a)

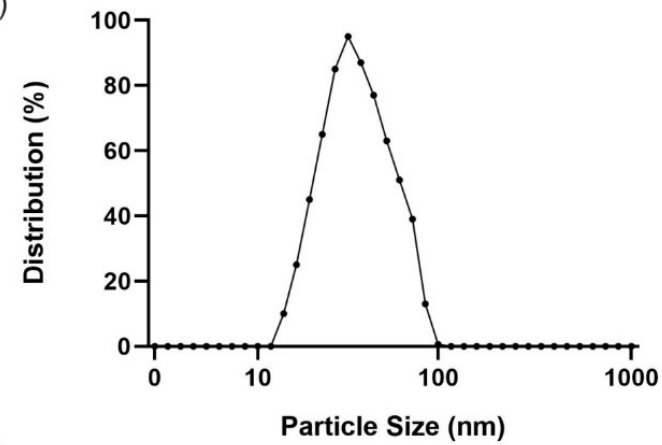

c)

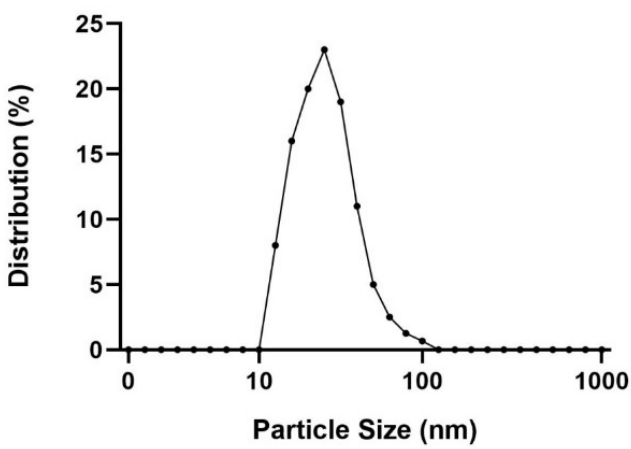

b)

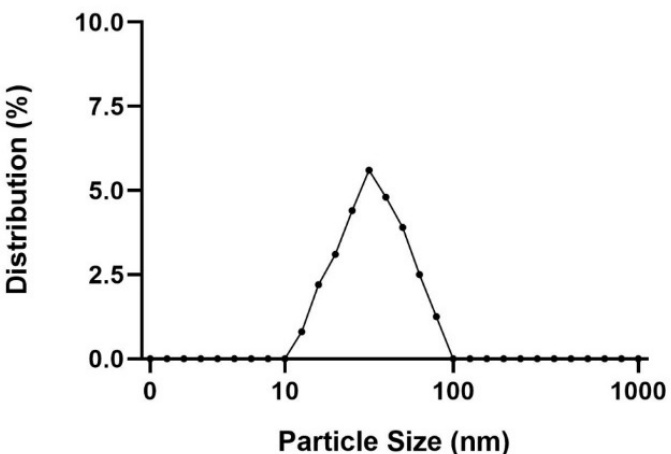

d)

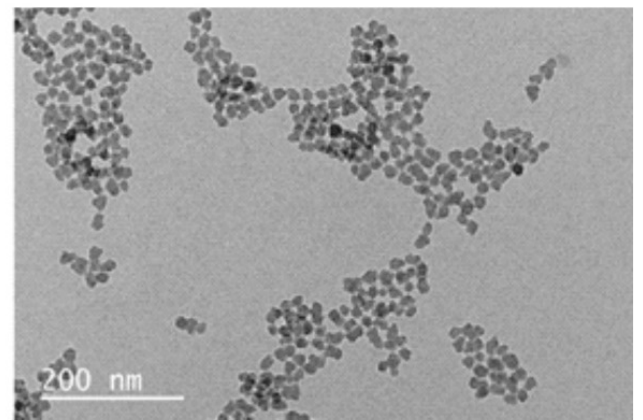

Figure I Characterization studies of nanoparticles a) AgNP Size Distribution Graphics, b) FeNP Size Distribution Graphics, c) SiNP Size Distribution Graphics, d) SiNPTEM Image.

a)

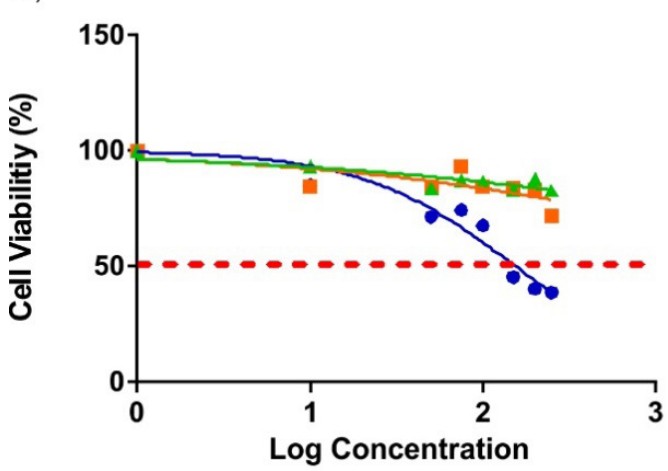

b)

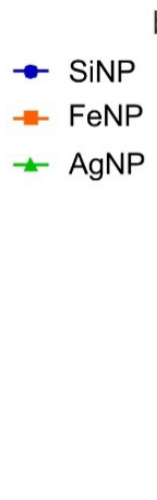

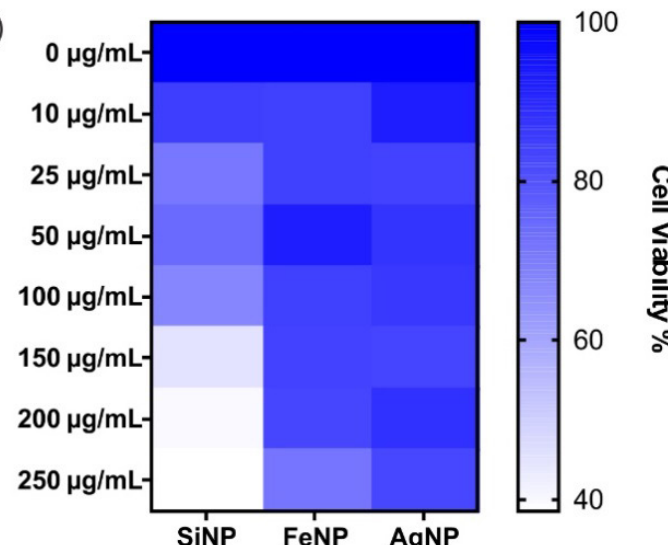

Figure 2 Results of cytotoxicity studies with A549 cells, a) logarithmic dose-dependent sigmoidal graph, and b) heat-map graph indicating percentage viability. 


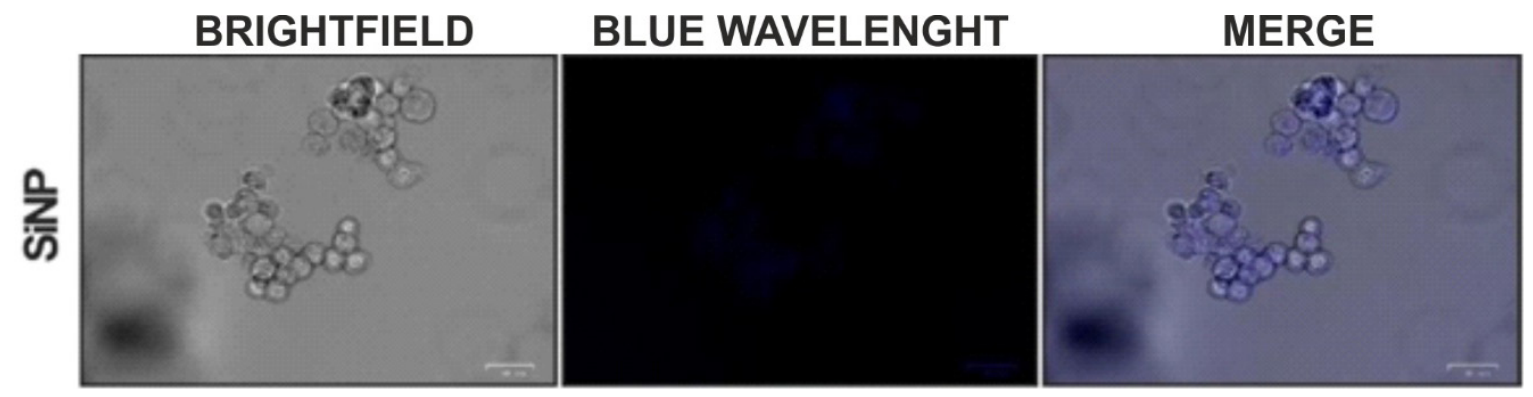

Figure 3 Brightfield, fluorescence and merge images of silica nanoparticles on a fluorescent cell imager.

The cytotoxic effect of AgNP and FeNP, which we used in our study, could not be observed in lung cancer cells, and it was concluded that only SiNP had a cytotoxic effect. It was observed that the size and toxicity of SiNP affect cancer cells inversely proportionally. Within the scope of our project, three different nanoparticle effects, whose chemical and biological activities were investigated, were used to treat the cancer type with the highest mortality today. In the treatment of nanoparticles of the same size but different material properties with A549 cells, the silica nanoparticle showed its effect at increasing concentrations and significantly affected the cell viability. Silica nanoparticles are a good alternative to conventional chemotherapy due to their large surface area and superior abilities in drug delivery systems.$^{36}$ Lin et al. (2006) determined that the cell's cytotoxic effect increased in a dose-dependent manner after being treated with $15 \mathrm{~nm}$ and $46 \mathrm{~nm}$ SiNPs in lung cancer cells. In a dose-dependent manner, $100 \mu \mathrm{g} / \mathrm{mL}$ concentrations of SiNPs decreased the viability of cells by $45.4 \%$ after 72 hours. In addition, while SiNPs increased the level of ROS in the cell, glutathione level was reduced. They also found that the cell membrane was damaged as a result of lipid peroxidation. ${ }^{37}$ When the studies and the data obtained are compared, it is also supported in the studies that the effectiveness of AgNP and FeNPs on cells with $\pm 40 \mathrm{~nm}$ dimensions is less. In SiNPs, the effects of studies conducted at $15-46 \mathrm{~nm}$ on lung cancer cells were supported by their cytotoxic and molecular effects. Therefore, it is seen that SiNP can be a drug candidate that can be used in an alternative and new treatment method to treat lung cancer. Advantages such as the nanoparticles used are non-toxic to other healthy cells, stable in the blood, and providing an uninterrupted effect on the cancerous cell depending on the variables in the tumor environment provides different approaches for new treatment methods. The fact that the iron nanoparticle does not show cytotoxicity shows that studies should be done to increase its efficiency structurally. Silica nanoparticles, in which we obtained a significant change in the cytotoxic effect, showed us that the cell viability decreased in a dose-dependent manner.

\section{Conclusion}

In the data obtained from literature studies, the effects of many different nanoparticles on different cancer cells were examined in various aspects. However, the cytotoxic effect of AgNP and FeNP we used in our study was not observed in lung cancer cells, and it was concluded that only SiNP had a cytotoxic effect. The functionalization of the surface of silica nanoparticles and the ability to bind functional groups or drug candidates both on the surface and inside make them an essential agent in cancer treatments.

\section{Acknowledgments}

This project founding by The Scientific and Technological Research Council of Turkey (TUBITAK) Grant No. 1919B01190248.

\section{Conflicts of interest}

Authors declare that there is no conflict of interest.

\section{References}

1. Global Burden of Disease Cancer C, Fitzmaurice C, Dicker D, Pain A, et al. The Global Burden of Cancer 2013. JAMA Oncol. 2015;1(4):505-527.

2. Sung H, Ferlay J, Siegel RL, et al. Global Cancer Statistics 2020: GLOBOCAN Estimates of Incidence and Mortality Worldwide for 36 Cancers in 185 Countries. CA Cancer J Clin. 2021;71(3):209-249.

3. Dias M, Linhas R, Campainha S, et al. Lung cancer in never-smokerswhat are the differences? Acta Oncol. 2017;56(7):931-935.

4. Yokota J, Wada M, Shimosato Y, et al. Loss of heterozygosity on chromosomes 3,13 , and 17 in small-cell carcinoma and on chromosome 3 in adenocarcinoma of the lung. Proc Natl Acad Sci U S A. 1987;84(24):9252-9256.

5. Buiatti E, Geddes M, Arniani S. Epidemiology of lung cancer. Ann Ist Super Sanita. 1996;32(1):133-144.

6. Singh KK. Nanotechnology in cancer detection and treatment. Technol Cancer Res Treat. 2005;4(6):583.

7. Yokoyama M. Drug targeting with nano-sized carrier systems. J Artif Organs. 2005;8(2):77-84.

8. Nahar M, Dutta T, Murugesan S, et al. Functional polymeric nanoparticles: an efficient and promising tool for active delivery of bioactives. Crit Rev Ther Drug Carrier Syst. 2006;23(4):259-318.

9. Zhang XF, Liu ZG, Shen W, et al. Silver Nanoparticles: Synthesis, Characterization, Properties, Applications, and Therapeutic Approaches. Int J Mol Sci. 2016;17(9):1534.

10. Bao G, Mitragotri S, Tong S. Multifunctional nanoparticles for drug delivery and molecular imaging. Аnпи Rev Biomed Eng. 2013;15:253-282.

11. Pugazhendhi A, Edison T, Karuppusamy I, et al. Inorganic nanoparticles: A potential cancer therapy for human welfare. Int J Pharm. 2018;539(12):104-111.

12. Sharma A, Goyal AK, Rath G. Recent advances in metal nanoparticles in cancer therapy. J Drug Target. 2018;26(8):617-632.

13. Kumar R, Chauhan A, Jha SK, et al. Encapsulated lanthanum strontium manganese oxide in mesoporous silica shell: Potential for cancer treatment by hyperthermia therapy. Journal of Alloys and Compounds. 2019;790:433-446.

14. Rao KS, El-Hami K, Kodaki T, et al. A novel method for synthesis of silica nanoparticles. Journal of Colloid and Interface Science. 2005;289(1):125-131.

15. Zhang J, Fu Y, Mei Y, et al. Fluorescent metal nanoshell probe to detect single miRNA in lung cancer cell. Analytical Chemistry. 2010;82(11):4464-4471. 
16. Rogers WJ, Basu P. Factors regulating macrophage endocytosis of nanoparticles: implications for targeted magnetic resonance plaque imaging. Atherosclerosis. 2005;178(1):67-73.

17. Coricovac DE, Moaca EA, Pinzaru I, et al. Biocompatible Colloida Suspensions Based on Magnetic Iron Oxide Nanoparticles: Synthesis, Characterization and Toxicological Profile. Front Pharmacol. 2017;8:154.

18. Soares PIP, Laia CAT, Carvalho A, et al. Iron oxide nanoparticles stabilized with a bilayer of oleic acid for magnetic hyperthermia and MRI applications. Applied Surface Science. 2016;383:240-247.

19. Cherukuri P, Glazer ES, Curley SA. Targeted hyperthermia using metal nanoparticles. Adv Drug Deliv Rev. 2010;62(3):339-345.

20. Bulte JW, Kraitchman DL. Iron oxide MR contrast agents for molecular and cellular imaging. NMR Biomed. 2004;17(7):484-499.

21. Durmus IM, Deveci I, Karakurt S. Synthesis of Silica Based Nanoparticles against the Proliferation of Human Prostate Cancer. Anticancer Agents Med Chem. 2021

22. Karakurt S, Kandir S, Gökçek-Saraç Ç. Upregulation of p53 by tannic acid treatment suppresses the proliferation of human colorectal carcinoma. Acta Pharmaceutica. 2021;71(4):587-602.

23. Baetke SC, Lammers T, Kiessling F. Applications of nanoparticles for diagnosis and therapy of cancer. Br J Radiol. 2015;88(1054):20150207.

24. Cryer AM, Thorley AJ. Nanotechnology in the diagnosis and treatment of lung cancer. Pharmacol Ther. 2019;198:189-205.

25. Carrasco-Esteban E, Dominguez-Rullan JA, Barrionuevo-Castillo P, et al. Current role of nanoparticles in the treatment of lung cancer. J Clin Transl Res. 2021;7(2):140-155.

26. Woodman C, Vundu G, George A, et al. Applications and strategies in nanodiagnosis and nanotherapy in lung cancer. Semin Cancer Biol. 2021;69:349-364.

27. Kandasamy G, Maity D. Recent advances in superparamagnetic iron oxide nanoparticles (SPIONs) for in vitro and in vivo cancer nanotheranostics. Int J Pharm. 2015;496(2):191-218.
28. Chauhan A, Jha KS, Kuanr BK. Synthesis and characterization of iron oxide nanoparticles (IONPs) and their cytotoxicity effects on lung epithelial carcinoma cells. AIP Conference Proceedings: American Institute of Physics. 2017;1832(1).

29. Reczynska K, Marszalek M, Zarzycki A, et al. Superparamagnetic Iron Oxide Nanoparticles Modified with Silica Layers as Potential Agents for Lung Cancer Treatment. Nanomaterials (Basel). 2020;10(6):1076.

30. Sau TK, Rogach AL, Jackel F, et al. Properties and applications of colloidal nonspherical noble metal nanoparticles. Adv Mater. 2010;22(16):1805-1825.

31. Sperling RA, Rivera Gil P, Zhang F, et al. Biological applications of gold nanoparticles. Chem Soc Rev. 2008;37(9):1896-1908.

32. Porcel E, Liehn S, Remita H, et al. Platinum nanoparticles: a promising material for future cancer therapy? Nanotechnology. 2010;21(8):85103.

33. Danhier F, Feron O, Preat V. To exploit the tumor microenvironment: Passive and active tumor targeting of nanocarriers for anti-cancer drug delivery. J Control Release. 2010;148(2):135-146.

34. He Y, Du Z, Ma S, et al. Effects of green-synthesized silver nanoparticles on lung cancer cells in vitro and grown as xenograft tumors in vivo. Int J Nanomedicine. 2016;11:1879-1887.

35. Gliga AR, Skoglund S, Wallinder IO, et al. Size-dependent cytotoxicity of silver nanoparticles in human lung cells: the role of cellular uptake, agglomeration and Ag release. Part Fibre Toxicol. 2014;11:11.

36. Gao Y, Gao D, Shen J, et al. A Review of Mesoporous Silica Nanoparticle Delivery Systems in Chemo-Based Combination Cancer Therapies. Front Chem. 2020;8:598722.

37. Lin W, Huang YW, Zhou XD, et al. In vitro toxicity of silica nanoparticles in human lung cancer cells. Toxicol Appl Pharmacol. 2006;217(3):252-259. 